\title{
"Connectomic surgery": diffusion tensor imaging (DTI) tractography as a targeting modality for surgical modulation of neural networks
}

\section{Jaimie M. Henderson *}

Neurosurgery, Stanford University School of Medicine, Stanford, CA, USA

Edited by:

John T. Gale, Cleveland Clinic, USA

Reviewed by:

Antonio Pereira, Federal University of Rio Grande do Norte, Brazil

Ken Sakaie, Cleveland Clinic, USA

\section{*Correspondence:}

Jaimie M. Henderson,

Neurosurgery, Stanford University

School of Medicine, 300 Pasteur

Dr., Edwards Bldg./R-227, Stanford,

CA 94305, USA.

e-mail: henderj@stanford.edu
Deep brain stimulation (DBS) is being used to treat a growing number of neurological disorders. Until recently, DBS has been thought to act mainly by suppressing local neuronal activity, essentially producing a functional lesion. Numerous studies are now demonstrating that DBS has widespread network effects mediated by white matter pathways. The new science of connectomics aims to map the connectivity between brain regions in health and disease. Targeting DBS specifically to pathways which exhibit pathological connectivity could greatly expand the possibilities for treating brain diseases. This brief review examines the current state of brain imaging for visualization of these networks and describes how DBS might be used to restore normal connectivity in pathological states.

Keywords: deep brain stimulation, connectomics, diffusion tensor imaging, default mode network, tractography

\section{INTRODUCTION}

Since its initial introduction in the early 1950s (Delgado et al., 1952), and its refinement in the late 1980s to its current modern form (Benabid et al., 1987), deep brain stimulation (DBS) has been increasingly employed in the treatment of neurodegenerative diseases. The safety, adjustability and reversibility of this modality have driven widespread adoption, essentially replacing brain lesioning for the treatment of movement disorders such as Parkinson's disease (Kleiner-Fisman et al., 2006; Weaver et al., 2009; Bronstein et al., 2011), tremor (Flora et al., 2010), and dystonia (Isaias et al., 2009; Krauss, 2010). The non-destructive nature of DBS has encouraged exploration as a treatment modality for psychiatric disorders such as depression and obsessivecompulsive disorder (Greenberg et al., 2008; Malone et al., 2009; Lozano et al., 2012), obesity (Tomycz et al., 2011), and memory disorders (Laxton et al., 2010), as well as its refinement in the treatment of pain (Bittar et al., 2005). Given the growing number of disorders being treated by DBS, increasing efforts have been dedicated to understanding its mechanism of action. Initially, DBS was felt to act by purely inhibitory influences, mimicking destruction of a brain nucleus (Breit et al., 2004). It is now generally accepted that DBS can have complex influences over widespread areas of the brain (Dostrovsky and Lozano, 2002; Johnson et al., 2008; Montgomery and Gale, 2008; Hauptmann and Tass, 2010; McIntyre and Hahn, 2010), which have implications beyond the straightforward inhibition of a local gray matter structure. DBS is no longer thought of as a simple substitute for lesioning, but is being appreciated as a means to modulate entire neural networks with increasing precision.

Functional imaging has allowed us to peer more deeply into the physiological state of the brain during health and disease and has been used to both guide DBS development (Mayberg et al., 2005) and to better elucidate its mechanisms (Kringelbach et al.,
2010). The purpose of this brief review is to examine the current state of knowledge about brain networks and their visualization with modern imaging modalities, and how this information can be used to better guide neurostimulation interventions in treating a broad range of neurological disorders.

\section{DIFFUSION TENSOR IMAGING AND TRACTOGRAPHY}

White matter tracts are formed from the axonal projections of cortical and subcortical neurons, creating the structural connective scaffolding of the brain. Using the recent technique of diffusion tensor imaging (DTI), these tracts can now be visualized in vivo. DTI takes advantage of the normal diffusion patterns of water molecules through the brain by Brownian motion. Each brain region has a distinct pattern of diffusion, limited by cell membranes, internal cellular structure, and other local factors such as myelin. Water molecules can be magnetically labeled by application of a strong magnetic gradient during magnetic resonance imaging (MRI). Differences in apparent water mobility determine the signal attenuation in response to these gradients, allowing the directionality of diffusion to be calculated for each voxel (Basser et al., 1994). Water mobility is assumed to be greatest along axon bundles. Given this assumption, white matter fascicles can be traced from voxel to voxel by following the direction of greatest water diffusion (Conturo et al., 1999; Mori et al., 1999; Basser et al., 2000). Jones' 2008 review (Jones, 2008) provides an excellent overview.

Although DTI tractography potentially suffers from a number of limitations (including poor resolution, inaccuracies introduced by poor signal-to-noise ratio, and possible mis-registration with anatomic images) (Mori and van Zijl, 2002), there is increasing evidence that this method provides useful information for locating white matter tracts in the brain. Establishing structural connectivity by this method is thus useful for studying 
structure-function relationships with functional imaging techniques. Anatomically, DTI tractography still cannot provide resolution comparable to direct fiber dissection in preserved human brains (Peltier et al., 2010) or chemical tract-tracing methods in animals (Lehman et al., 2011). However, stimulation of tractographically defined white matter connections has been successfully employed as a technique for avoiding complications in neurosurgery (Berman et al., 2007; Coenen et al., 2009; Bello et al., 2010; Leclercq et al., 2010), suggesting its utility for guiding therapeutic brain stimulation. Insights from animal studies are already helping to direct DTI tractography studies of DBS mechanisms in Parkinson's disease (Gradinaru et al., 2009) and mood disorders (Lehman et al., 2011).

\section{THE "CONNECTOME" CONCEPT OF BRAIN FUNCTION}

Over the past decade, a number of technologies have converged to make large-scale mapping of the brain possible. With the advent of more advanced imaging methods and the increasing speed and storage capacity of computers, it is now feasible to create large databases of functional and structural connectivity, in a way similar to that pursued for the human genome. The resulting field of "connectomics" aims to map the structure of the brain at several levels from individual neurons to nodes and networks, and to understand how that structure gives rise to the complex functions of the brain (Hagmann et al., 2010).

The relationship between structural connectivity (as demonstrated by physical tract-tracing by chemical or anatomical means, or by imaging methods such as DTI tractography) and functional connectivity [as demonstrated by functional MRI (fMRI), positron emission tomography (PET), or electrophysiological measurements] is dynamic over multiple time scales. Several studies have examined connectivity in the brain's resting state (the so-called "default mode network"), demonstrating general concordance between structural and functional connectivity while acknowledging that the correspondence is not absolute (Damoiseaux and Greicius, 2009). Functional connectivity without demonstrated structural connectivity could occur through indirect connections, and it is possible for functional connectivity to change dynamically during task performance even if structural connectivity remains constant (Hampson et al., 2004; Damoiseaux and Greicius, 2009).

While specific brain regions have long been known to subserve specific functions, there is a growing realization that the brain is far less rigidly organized than previously thought. The modern view of brain architecture is that of a flexible, re-configurable network organized into connected modules (Deco et al., 2008; De Benedictis and Duffau, 2011; Zamora-López et al., 2011). Increasingly, the connectivity between brain regions is being seen as dynamic on time scales ranging from seconds (Honey et al., 2007) to years (Uddin et al., 2011), organized around a structural modular scaffolding (Deco et al., 2011). This ever-changing neuronal landscape allows for the flexibility in behavior and thought that is the hallmark of human cognition.

Neural connectivity varies depending on the scale at which it is examined (Deco et al., 2008). At the microscopic scale (i.e., a cortical slice or subcortical gray matter), neurons are densely and homogeneously connected with one another. At larger scales, neuronal ensembles are assembled into less densely connected assemblies such as cortical columns (Mountcastle, 1957). At the scale of the entire brain, white matter tracts sparsely connect these neural ensembles one to another. These large-scale connections between functional units are potential targets for modulation by methods such as DBS.

Specialization of function requires that neuronal pathways remain segregated, but integration of sensory, motor, cognitive, and emotional inputs is required for decision-making at the organismal level. How does the brain segregate and integrate simultaneously? There is still considerable debate regarding whether integration occurs at topographically localized regions (space) or emerges as a consequence of synchronized neuronal activity (time). However, in either case, functional connectivity between regions is likely established via "hubs" in the neural network, representing more richly interconnected areas in the cortex or subcortical nuclei (Zamora-López et al., 2011).

Many of the subcortical structures that have been targeted for the surgical treatment of movement disorders show dense, widespread connectivity, and may represent this kind of integrative hub within an overall less densely connected network. Disruption of integration or segregation within these hubs could lead to abnormalities in connectivity, creating pathological brain states. For example, several studies have demonstrated increased connectivity in Parkinson's disease, using resting-state fMRI (Baudrexel et al., 2011), task-related fMRI (Wu et al., 2010) or PET (Poston and Eidelberg, 2011). In the resting state, increased connectivity between cortex and the subthalamic nucleus (STN) (Baudrexel et al., 2011) mirrors the abnormal electrical synchrony in the beta $(13-35 \mathrm{~Hz})$ frequency band, which has been hypothesized to play a role in the generation of Parkinsonian symptoms (Brown et al., 1998; Bronte-Stewart et al., 2009; de Solages et al., 2011). De-synchronization of pathologically synchronized circuits, with consequent normalization of functional connectivity, could therefore represent an underlying theme in DBS mechanisms (Hauptmann and Tass, 2010).

\section{WHITE MATTER AS THE MAIN TARGET OF DEEP BRAIN STIMULATION}

It has been known since the 1960s that white matter tracts are, in general, more sensitive to electrical stimulation than cell bodies (Landau et al., 1965). Numerous studies since that time have contributed to the notion that axons are the primary neuronal elements acted on by electrical stimulation (McIntyre et al., 2004; Butson et al., 2007; Gradinaru et al., 2009; Deniau et al., 2010). It seems increasingly likely that DBS affects distant as well as local network nodes, propagated by stimulation of white matter (Deniau et al., 2010). For example, high frequency stimulation of the subthalamic region has been shown to cause physiological effects in numerous nuclei connected to the STN, including SNR, GP, SNpc, striatum, contralateral STN, PPN, thalamus, cortex, and superior colliculus (McIntyre and Hahn, 2010). A study examining the connectivity of two different deep brain targets for depression-the subcallosal cingulate region and the anterior limb of the internal capsule-revealed largely non-overlapping projection patterns, with the subcallosal cingulate projecting to 
more medial-limbic-striatal regions and the anterior limb of the internal capsule projecting to more lateral cortical-thalamictemporal regions (Gutman et al., 2009). The widespread connectivity of these regions has been verified by anatomical tracing methods in animals (Lehman et al., 2011). All forms of therapeutic electrical brain stimulation have been found to cause changes in activity throughout widespread networks which differ for each area, as measured by functional imaging techniques (McIntyre and Hahn, 2010).

Although DBS of the subthalamic region for Parkinson's disease has been previously hypothesized to act by suppressing local activity, optogenetic experiments in laboratory animals suggest that antidromic activation of the "hyperdirect" pathway from motor areas to the STN may also play a prominent role (Gradinaru et al., 2009). In a series of patients undergoing subthalamic DBS for Parkinson's disease, this hypothesis was tested by recording from the cortical surface, at a location defined by DTI tractography as structurally connected with the STN. The results, to be published in a separate study, demonstrate functional electrical connectivity between these structurally connected regions, corroborating the findings from functional imaging studies (Figure 1).
Given the growing body of evidence implicating white matter as the main target for DBS, direct tract targeting guided by DTI might be hypothesized to provide efficacy equivalent to, or even possibly better than, targeting of nuclei. In a patient with Parkinson's disease undergoing thalamic DBS for tremor, Coenen and colleagues demonstrated, using image fusion of postoperative lead position and preoperative DTI imaging, that the dentatorubrothalamic tract (DRT) was included within the area influenced by the electrical field (Coenen et al., 2011b). Noting that the DRT has long been hypothesized to play an important role in the neurosurgical control of tremor, his group went on to directly target this tract with good symptomatic results (Coenen et al., 2011a). Several studies have suggested that the posterior subthalamic area or the caudal zona inserta may be superior as a target for tremor (Plaha et al., 2004; Hamel et al., 2007; Sandvik et al., 2011; Coenen et al., 2011b). Given the course of the DRT, it is likely that all of these targets (posterior subthalamic region, caudal zona incerta, and Vim nucleus of the thalamus) lie along the same white matter pathway. In fact, deviation from this tractographically defined target (as may occur during electrophysiologically guided DBS of the Vim nucleus of the thalamus, the traditional target), can lead to poor tremor

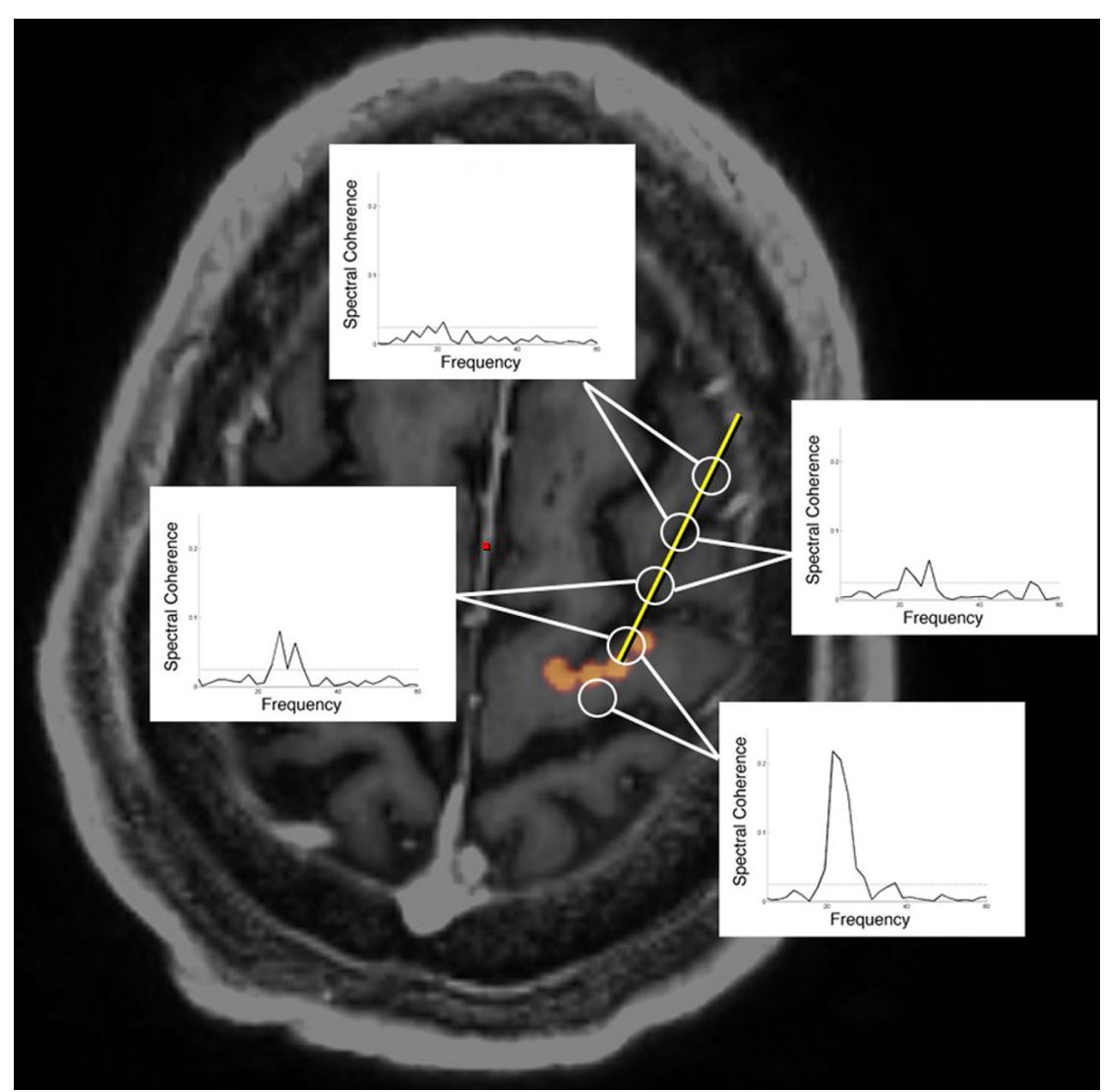

FIGURE 1 | Oscillatory coherence demonstrated between cortex and subthalamic nucleus during DBS surgery. A six contact cortical strip recording electrode (open circles) was introduced through a $14 \mathrm{~mm}$ burrhole to arrive at the origin of the hyperdirect pathway (denoted in orange) as defined by DTI tractography. Electrocorticographic recordings demonstrate oscillatory coherence at $22 \mathrm{~Hz}$ ("beta" frequency range), most strongly between the subthalamic nucleus (STN) and the pair of electrodes straddling the origin of the hyperdirect pathway (Henderson et al., 2010). 


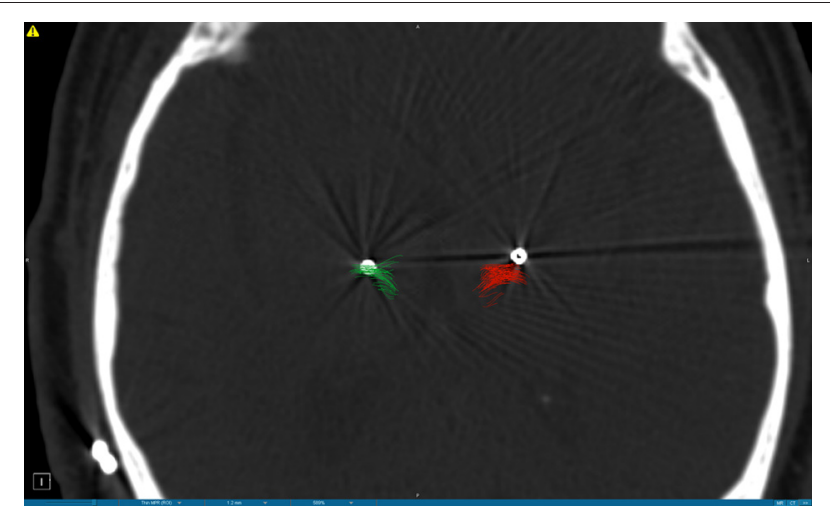

FIGURE 2 | Postoperative CT scan demonstrating deep brain stimulation electrodes placed in the Vim nucleus of the thalamus for the treatment of tremor. DTI tractography of the dentatorubrothalamic tract (DRT) is superimposed on the CT scan, and is indicated in green on the left side (corresponding to the radiographic right side of the brain) and in red on the right side (radiographic left side of the brain). Note the position of the DBS lead on the right side of the brain lies within the DRT and provided symptomatic relief, whereas the DBS lead on the left side of the brain is located outside the DRT and did not provide symptomatic relief.

control, as demonstrated in a patient with bilateral DBS for essential tremor (Figure 2).

\section{NETWORK SURGERY: MODULATION OF WIDESPREAD CONNECTIONS IN THE MINIMALLY CONSCIOUS STATE}

Following severe brain injury, some patients may show waxing and waning of consciousness with relative preservation of integrative brain networks in what is called the minimally conscious state (MCS), characterized by intermittent awareness of self and surroundings (Schiff et al., 2007). This state is hypothesized to reflect a localized lesion to the ascending systems responsible for arousal, with sparing of cortical integrative circuitry. The central thalamus receives ascending connections from brainstem "arousal centers" and sends projections to widespread regions of the forebrain. Disconnection from these deeper centers may produce decreased resting tone throughout the cortex (Schiff, 2009). A study of resting state connectivity using fMRI in patients with disordered consciousness found reduced functional connectivity in posterior cingulate cortex, medial prefrontal cortex, temporoparietal regions, and parahippocampal gyrus in patients in the MCS vs. normal, healthy controls (Vanhaudenhuyse et al., 2010).

The central thalamic nuclear group thus likely represents an important network hub, which might serve as a target for neuromodulation to address deficits in attention and/or arousal (Schiff, 2009). In 2007, Schiff and colleagues reported on a patient in the MCS who improved with DBS of the central thalamus, including the anterior intralaminar thalamic nuclei and adjacent paralaminar regions of thalamic association nuclei (Schiff et al., 2007). During active DBS, the study participant demonstrated increased periods of arousal, functional object use and intelligible verbalization (which had not been present before). The authors hypothesized that this participant suffered from underactivation of cortical networks subserving consciousness with relatively preserved structural connectivity, and that DBS served to activate these connected regions. The necessity of maintained structural integrity in the face of functional loss is suggested by a similar study which was carried out in a large number of patients in a persistent vegetative state, without success (Schiff, 2009). Presumably, these patients lacked the structural connectivity necessary to produce widespread activation and increased arousal with central thalamic stimulation.

DBS for the MCS is thus aimed purposely at activating largescale networks throughout the brain, rather than inactivating a single localized area. The initial success of this modality adds yet more evidence suggesting that network effects may dominate over local effects in therapeutic DBS.

\section{CONNECTOMICS AND CURRENT DBS TARGETS}

In light of the foregoing evidence, it is clear that current DBS targets share several features, including their location along white matter pathways and their influence on distributed networks, which allow them to be naturally considered within the framework of connectomics. Stimulation of the STN for Parkinson's disease affects distant targets through both antidromic and orthodromic projections (Gradinaru et al., 2009; McIntyre and Hahn, 2010), and may exert its therapeutic effect by normalizing functional connectivity. Several effective DBS targets for tremor lie along the DRT, suggesting that they are all modulating the same structural pathway to achieve a similar functional result (Coenen et al., 2011a). DBS of the subgenual cingulate region acts by changing not only local metabolic activity, but also the activity of functionally connected distant targets (Mayberg et al., 2005). Thalamic DBS for treatment of the MCS activates widespread areas of cortex by virtue of their connectivity with the target site in the central thalamus (Schiff et al., 2007; Schiff, 2008). Finally, DBS of the fornix for the treatment of memory disorders is specifically targeted at a white matter structure which connects directly to the hippocampus, long known to be intimately involved with memory storage and retrieval (Laxton et al., 2010).

\section{CONCLUSIONS}

There is growing evidence that stimulation of white matter tracts connecting functional hubs within brain networks can restore normal patterns of activity in widespread brain regions. Although suggested by preliminary studies, it remains to be definitively demonstrated whether these modulatory influences are due to changes in connectivity, and whether connectivity normalization can be related to other markers such as oscillatory synchronization or frequency of DBS. However, the suggestion that altered functional connectivity is a hallmark of many brain diseases, and that normalizing abnormal connectivity with electrical stimulation can lead to symptomatic improvement, introduces a new and exciting way of thinking about DBS (Kringelbach et al., 2011).

To fully evaluate the potential of "connectomic surgery," a number of challenges remain to be met. There is a need for further refinement of DTI tractography methods, particularly with respect to improving signal-to-noise ratio, reducing voxel size, reducing distortion with the consequence of better co-registration with anatomic MR, and the development of better techniques for dealing with crossing fibers and multiple fiber directions. 
There is also a need to refine methods of reliably relating structural connectivity to functional connectivity, since DBS requires an intact structural pathway to modulate. Connectomic research has made great strides in a few short years, but there are still large gaps in connectomic databases which will require continued effort to fill (Hagmann et al., 2010). DBS electrodes and stimulation paradigms could be better optimized for tract stimulation as opposed to introduction into deep brain nuclei. Finally, the way of thinking about brain diseases amenable to DBS treatment may need to be altered. Approaching further study of brain network

\section{REFERENCES}

Basser, P. J., Mattiello, J., and LeBihan, D. (1994). MR diffusion tensor spectroscopy and imaging. Biophys. J. 66, 259.

Basser, P. J., Pajevic, S., Pierpaoli, C., Duda, J., and Aldroubi, A. (2000). In vivo fiber tractography using DT-MRI data. Magn. Reson. Med. 44, 625-632.

Baudrexel, S., Witte, T., Seifried, C., von Wegner, F., Beissner, F., Klein, J. C., Steinmetz, H., Deichmann, R., Roeper, J., and Hilker, R. (2011). Resting state fMRI reveals increased subthalamic nucleus-motor cortex connectivity in Parkinson's disease. Neuroimage 55, 1728-1738.

Bello, L., Castellano, A., Fava, E., Casaceli, G., Riva, M., Scotti, G., Gaini, S. M., and Falini, A. (2010). Intraoperative use of diffusion tensor imaging fiber tractography and subcortical mapping for resection of gliomas: technical considerations. Neurosurg. Focus 28, E6.

Benabid, A. L., Pollak, P., Louveau, A., Henry, S., and de Rougemont, J. (1987). Combined (thalamotomy and stimulation) stereotactic surgery of the VIM thalamic nucleus for bilateral Parkinson disease. Appl. Neurophysiol. 50, 344-346.

Berman, J. I., Berger, M. S., Chung, S. W., Nagarajan, S. S., and Henry, R. G. (2007). Accuracy of diffusion tensor magnetic resonance imaging tractography assessed using intraoperative subcortical stimulation mapping and magnetic source imaging. J. Neurosurg. 107, 488-494.

Bittar, R. G., Kar-Purkayastha, I., Owen, S. L., Bear, R. E., Green, A., Wang, S., and Aziz, T. Z. (2005). Deep brain stimulation for pain relief: a meta-analysis. J. Clin. Neurosci. 12, 515-519.

Breit, S., Schulz, J. R. B., and Benabid, A.-L. (2004). Deep brain stimulation. Cell Tissue Res. 318, 275-288.

Bronstein, J. M., Tagliati, M., Alterman, R. L., Lozano, A. M., Volkmann, J., Stefani, A., Horak, F. B., Okun, M. S., Foote, K. D., Krack, P., Pahwa,
R., Henderson, J. M., Hariz, M. I., Bakay, R. A., Rezai, A., Marks, W. J., Moro, E., Vitek, J. L., Weaver, F. M., Gross, R. E., and DeLong, M. R. (2011). Deep brain stimulation for Parkinson disease: an expert consensus and review of key issues. Arch. Neurol. 68, 165-171. Koop, M. M., Hill, B. C., Henderson, J. M., and Wingeier, B. (2009). The STN beta-band profile in Parkinson's disease is stationary and shows prolonged attenuation after deep brain stimulation. Exp. Neurol. 215, 20-28.

Brown, E. N., Frank, L. M., Tang, D., Quirk, M. C., and Wilson, M. A. (1998). A statistical paradigm for neural spike train decoding applied to position prediction from the ensemble firing patterns of rat hippocampal place cells. J. Neurosci. 18, 7411-7425.

Butson, C. R., Cooper, S. E., Henderson, J. M., and McIntyre, C. C. (2007). Patient-specific analysis of the volume of tissue activated during deep brain stimulation. Neuroimage 34, 661-670.

Coenen, V. A., Allert, N., and Mädler, B. (2011a). A role of diffusion tensor imaging fiber tracking in DBS of the dentato-rubro-thalamic tract (drt) for the treatment of therapy-refractory tremor. Acta Neurochir. (Wien) 153, 1579-1585; discussion 1585

Coenen, V. A., Honey, C. R., Hurwitz, T., Rahman, A. A., McMaster, J., Bürgel, U., and Mädler, B. (2009). Medial forebrain bundle stimulation as a pathophysiological mechanism for hypomania in subthalamic nucleus deep brain stimulation for Parkinson's disease. Neurosurgery 64, 1106-1114; discussion 1114-1115.

Coenen, V. A., Mädler, B., Schiffbauer, H., Urbach, H., and Allert, N. (2011b). Individual fiber anatomy of the subthalamic region revealed with diffusion tensor imaging: a concept to identify the deep
Bronte-Stewart, H., Barberini, C., deep brain stimulation surgery:

abnormalities with an eye toward neuromodulatory therapies might provide new insights which could guide future efforts.

In the same way that sequencing of the genome has already led to advances in the understanding of disease states, further definition of the connectome of the human brain could allow individualized connectivity mapping, perhaps leading to patientspecific maps of dysfunction in disease states (Hagmann et al., 2010). If DBS can indeed reliably alter functional connectivity, it could be a powerful tool for leveraging the insights of connectomics for the treatment of neurological disease.

brain stimulation target for tremor suppression. Neurosurgery 68 1069-1075; discussion 1075-1076.

Conturo, T. E., Lori, N. F., Cull, T. S., Akbudak, E., Snyder, A. Z., Shimony, J. S., McKinstry, R. C., Burton, H., and Raichle, M E. (1999). Tracking neuronal fiber pathways in the living human brain. Proc. Natl. Acad. Sci. U.S.A. 96, 10422-10427.

Damoiseaux, J. S., and Greicius, M. D (2009). Greater than the sum of its parts: a review of studies combining structural connectivity and restingstate functional connectivity. Brain Struct. Funct. 213, 525-533.

De Benedictis, A., and Duffau, $H$. (2011). Brain hodotopy: from esoteric concept to practical surgical applications. Neurosurgery 68, 1709-1723; discussion 1723.

de Solages, C., Hill, B. C., Yu, H., Henderson, J. M., and BronteStewart, H. (2011). Maximal subthalamic beta hypersynchrony of the local field potential in Parkinson's disease is located in the central region of the nucleus. J. Neurol. Neurosurg. Psychiatry 82, 1387-1389.

Deco, G., Jirsa, V. K., and McIntosh, A. R. (2011). Emerging concepts for the dynamical organization of resting-state activity in the brain. Nat. Rev. Neurosci. 12, 43-56.

Deco, G., Jirsa, V. K., Robinson, P. A., Breakspear, M., and Friston, K. (2008). The dynamic brain: from spiking neurons to neural masses and cortical fields. PLoS Comput. Biol. 4:e1000092. doi: 10.1371/journal.pcbi.1000092

Delgado, J. M., Hamlin, H., and Chapman, W. P. (1952). Technique of intracranial electrode implacement for recording and stimulation and its possible therapeutic value in psychotic patients. Confin. Neurol. 12, 315-319.

Deniau, J., Degos, B., Bosch, C., and Maurice, N. (2010). Deep brain stimulation mechanisms: beyond the concept of local functional inhibition. Eur. J. Neurosci. 32, 1080-1091.
Dostrovsky, J. O., and Lozano, A. M. (2002). Mechanisms of deep brain stimulation. Mov. Disord. 17 (Suppl. 3), S63-S68.

Flora, E. D., Perera, C. L., Cameron, A. L., and Maddern, G. J. (2010). Deep brain stimulation for essential tremor: a systematic review. Mov. Disord. 25, 1550-1559.

Gradinaru, V., Mogri, M., Thompson, K. R., Henderson, J. M., and Deisseroth, K. (2009). Optical deconstruction of parkinsonian neural circuitry. Science 324 , 354-359.

Greenberg, B. D., Gabriels, L. A., Malone, D. A., Rezai, A. R., Friehs, G. M., Okun, M. S., Shapira, N. A., Foote, K. D., Cosyns, P. R., Kubu, C. S., Malloy, P. F., Salloway, S. P., Giftakis, J. E., Rise, M. T., Machado, A. G., Baker, K. B., Stypulkowski, P. H., Goodman, W. K., Rasmussen, S. A., and Nuttin, B. J. (2008). Deep brain stimulation of the ventral internal capsule/ventral striatum for obsessive-compulsive disorder: worldwide experience. Mol. Psychiatry 15, 64-79.

Gutman, D. A., Holtzheimer, P. E., Behrens, T. E. J., Johansen-Berg, H., and Mayberg, H. S. (2009). A tractography analysis of two deep brain stimulation white matter targets for depression. Biol. Psychiatry 65, 276-282.

Hagmann, P., Cammoun, L., Gigandet, X., Gerhard, S., Grant, P. E., Wedeen, V., Meuli, R., Thiran, J-P., Honey, C. J., and Sporns, O. (2010). MR connectomics: principles and challenges. J. Neurosci. Methods 194, 34-45.

Hamel, W., Herzog, J., Kopper, F. Pinsker, M., Weinert, D., Müller, D., Krack, P., Deuschl, G., and Mehdorn, H. M. (2007). Deep brain stimulation in the subthalamic area is more effective than nucleus ventralis intermedius stimulation for bilateral intention tremor. Acta Neurochir. (Wien) 149, 749-758; discussion 758 .

Hampson, M., Olson, I. R., Leung, H.C., Skudlarski, P., and Gore, J. C. 
(2004). Changes in functional connectivity of human MT/V5 with visual motion input. Neuroreport $15,1315-1319$.

Hauptmann, C., and Tass, P. A. (2010). Restoration of segregated, physiological neuronal connectivity by desynchronizing stimulation. $J$. Neural Eng. 7, 056008.

Henderson, J. M., Bronte-Stewart, H., de Solages, C., Hill, B. C., and Atlas, S. W. (2010). "The human 'hyperdirect' pathway: diffusion tensor imaging tractography with physiological confirmation in Parkinson's disease patients," in Society for Neuroscience 40th Annual Meeting. San Diego, CA.

Honey, C. J., Kötter, R., Breakspear, M., and Sporns, O. (2007). Network structure of cerebral cortex shapes functional connectivity on multiple time scales. Proc. Natl. Acad. Sci. U.S.A. 104, 10240-10245.

Isaias, I. U., Alterman, R. L., and Tagliati, M. (2009). Deep brain stimulation for primary generalized dystonia: long-term outcomes. Arch. Neurol. 66, 465-470.

Johnson, M. D., Miocinovic, S., McIntyre, C. C., and Vitek, J. L. (2008). Mechanisms and targets of deep brain stimulation in movement disorders. Neurotherapeutics 5, 294-308.

Jones, D. K. (2008). Studying connections in the living human brain with diffusion MRI. Cortex 44, 936-952.

Kleiner-Fisman, G., Herzog, J., Fisman, D. N., Tamma, F., Lyons, K. E., Pahwa, R., Lang, A. E., and Deuschl, G. (2006). Subthalamic nucleus deep brain stimulation: summary and meta-analysis of outcomes. Mov. Disord. 21, S290-S304.

Krauss, J. K. (2010). Surgical treatment of dystonia. Eur. J. Neurol. 17 (Suppl. 1), 97-101.

Kringelbach, M. L., Green, A. L., and Aziz, T. Z. (2011). Balancing the brain: resting state networks and deep brain stimulation. Front. Integr. Neurosci. 5:8. doi: $10.3389 /$ fnint.2011.00008

Kringelbach, M. L., Green, A. L., Owen, S. L. F., Schweder, P. M., and Aziz, T. Z. (2010). Sing the mind electric principles of deep brain stimulation. Eur. J. Neurosci. 32, 1070-1079.

Landau, W. M., Bishop, G. H., and Clare, M. H. (1965). Site of excitation in stimulation of the motor cortex. J. Neurophysiol. 28, 1206-1222.

Laxton, A. W., Tang-Wai, D. F., McAndrews, M. P., Zumsteg, D., Wennberg, R., Keren, R., Wherrett,
J., Naglie, G., Hamani, C., Smith, G. S., and Lozano, A. M. (2010). A phase I trial of deep brain stimulation of memory circuits in Alzheimer's disease. Ann. Neurol. 68, 521-534.

Leclercq, D., Duffau, H., Delmaire, C., Capelle, L., Gatignol, P., Ducros, M., Chiras, J., and Lehéricy, S. (2010). Comparison of diffusion tensor imaging tractography of language tracts and intraoperative subcortical stimulations. J. Neurosurg. $112,503-511$.

Lehman, J. F., Greenberg, B. D., McIntyre, C. C., Rasmussen, S. A., and Haber, S. N. (2011). Rules ventral prefrontal cortical axons use to reach their targets: implications for diffusion tensor imaging tractography and deep brain stimulation for psychiatric illness. J. Neurosci. 31, 10392-10402.

Lozano, A. M., Giacobbe, P., Hamani, C., Rizvi, S. J., Kennedy, S. H., Kolivakis, T. T., Debonnel, G., Sadikot, A. F., Lam, R. W., Howard, A. K., Ilcewicz-Klimek, M., Honey, C. R., and Mayberg, H. S. (2012). A multicenter pilot study of subcallosal cingulate area deep brain stimulation for treatment-resistant depression. J. Neurosurg. 116, 315-322.

Malone, D. A., Dougherty, D. D., Rezai, A. R., Carpenter, L. L., Friehs, G. M., Eskandar, E. N., Rauch, S. L., Rasmussen, S. A., Machado, A. G., Kubu, C. S., Tyrka, A. R., Price, L. H., Stypulkowski, P. H., Giftakis, J. E., Rise, M. T., Malloy, P. F., Salloway, S. P., and Greenberg, B. D. (2009). Deep brain stimulation of the ventral capsule/ventral striatum for treatment-resistant depression. Biol. Psychiatry 65, 267-275.

Mayberg, H. S., Lozano, A. M., Voon, V., McNeely, H. E., Seminowicz, D., Hamani, C., Schwalb, J. M., and Kennedy, S. H. (2005). Deep brain stimulation for treatmentresistant depression. Neuron 45, 651-660.

McIntyre, C. C., and Hahn, P. J. (2010). Network perspectives on the mechanisms of deep brain stimulation. Neurobiol. Dis. 38, 329-337.

McIntyre, C. C., Grill, W. M., Sherman, D. L., and Thakor, N. V. (2004). Cellular effects of deep brain stimulation: model-based analysis of activation and inhibition. $J$. Neurophysiol. 91, 1457-1469.

Montgomery, E. B., and Gale, J. T. (2008). Mechanisms of action of deep brain stimulation(DBS). Neurosci. Biobehav. Rev. 32, 388-407.

Mori, S., Crain, B. J., Chacko, V., and van Zijl, P. (1999). Three-dimensional tracking of axonal projections in the brain by magnetic resonance imaging. Ann. Neurol. 45, 265-269.

Mori, S., and van Zijl, P. C. M. (2002). Fiber tracking: principles and strategies - a technical review. NMR Biomed. 15, 468-480.

Mountcastle, V. B. (1957). Modality and topographic properties of single neurons of cat's somatic sensory cortex. J. Neurophysiol. 20, 408-434.

Peltier, J., Verclytte, S., Delmaire, C., Pruvo, J.-P., Godefroy, O., and Le Gars, D. (2010). Microsurgical anatomy of the temporal stem: clinical relevance and correlations with diffusion tensor imaging fiber tracking. J. Neurosurg. 112, 1033-1038.

Plaha, P., Patel, N. K., and Gill, S. S. (2004). Stimulation of the subthalamic region for essential tremor. J. Neurosurg. 101, 48-54.

Poston, K. L., and Eidelberg, D. (2011). Functional brain networks and abnormal connectivity in the movement disorders. Neuroimage. [Epub ahead of print].

Sandvik, U., Koskinen, L.-O., Lundquist, A., and Blomstedt, P. (2011). Thalamic and subthalamic DBS for essential tremor: where is the optimal target? Neurosurgery $70,840-846$.

Schiff, N. (2009). Central thalamic deep-brain stimulation in the severely injured brain. Ann. N.Y. Acad. Sci. 1157, 101-116.

Schiff, N. D. (2008). Central thalamic contributions to arousal regulation and neurological disorders of consciousness. Ann. N.Y. Acad. Sci. 1129, 105-118.

Schiff, N. D., Giacino, J. T., Kalmar K., Victor, J. D., Baker, K., Gerber M., Fritz, B., Eisenberg, B., Biondi, T., O'Connor, J., Kobylarz, E. J. Farris, S., Machado, A., McCagg, C., Plum, F., Fins, J. J., and Rezai, A. R. (2007). Behavioural improvements with thalamic stimulation after severe traumatic brain injury. Nature 448, 600-603.

Tomycz, N. D., Whiting, D. M., and Oh, M. Y. (2011). Deep brain stimulation for obesity - from theoretical foundations to designing the first human pilot study. Neurosurg. Rev. 35, 37-43.

Uddin, L. Q., Supekar, K. S., Ryali, S., and Menon, V. (2011). Dynamic reconfiguration of structural and functional connectivity across core neurocognitive brain networks with development. J. Neurosci. 31, 18578-18589.

Vanhaudenhuyse, A., Noirhomme, Q., Tshibanda, L. J. F., Bruno, M. A., Boveroux, P., Schnakers, C. Soddu, A., Perlbarg, V., Ledoux, D., Brichant, J. F., Moonen, G., Maquet, P., Greicius, M. D., Laureys, S., and Boly, M. (2010). Default network connectivity reflects the level of consciousness in non-communicative brain-damaged patients. Brain 133, 161-171.

Weaver, F. M., Follett, K., Stern, M., Hur, K., Harris, C., Marks, W. J., Rothlind, J., Sagher, O., Reda, D., Moy, C. S., Pahwa, R., Burchiel, K., Hogarth, P., Lai, E. C., Duda, J. E., Holloway, K., Samii, A. Horn, S., Bronstein, J., Stoner, G., Heemskerk, J., and Huang, G. D. (2009). Bilateral deep brain stimulation vs. best medical therapy for patients with advanced Parkinson disease: a randomized controlled trial. JAMA 301, 63-73.

Wu, T., Chan, P., and Hallett, M. (2010). Effective connectivity of neural networks in automatic movements in Parkinson's disease. Neuroimage 49, 2581-2587.

Zamora-López, G., Zhou, C., and Kurths, J. (2011). Exploring brain function from anatomical connectivity. Front. Neurosci. 5:83. doi: 10.3389/fnins.2011.00083

Conflict of Interest Statement: The author declares that the research was conducted in the absence of any commercial or financial relationships that could be construed as a potential conflict of interest.

Received: 23 February 2012; paper pending published: 19 March 2012; accepted: 03 April 2012; published online: 24 April 2012.

Citation: Henderson JM (2012) "Connectomic surgery": diffusion tensor imaging (DTI) tractography as a targeting modality for surgical modulation of neural networks. Front. Integr. Neurosci. 6:15. doi: 10.3389/ fnint.2012.00015

Copyright (c) 2012 Henderson. This is an open-access article distributed under the terms of the Creative Commons Attribution Non Commercial License, which permits non-commercial use, distribution, and reproduction in other forums, provided the original authors and source are credited. 